\title{
Head and neck cancer: effective prevention in youth and predictive diagnostics for personalised treatment strategies according to biological differences
}

\author{
Andreas Dietz • Gunnar Wichmann
}

Received: 11 April 2011 / Accepted: 27 April 2011 /Published online: 14 May 2011

(C) European Association for Predictive, Preventive and Personalised Medicine 2011

\begin{abstract}
This article focuses on squamous cell carcinomas of the larynx, pharynx and oral cavity which count nearly $90 \%$ of the head and neck squamous cell carcinomas (HNSCC). Individual susceptibility depends on an individually given genetic background and/or acquired conditions or factors elevating the risk of HNSCC including smoking, alcohol abuse, and improper oral hygiene. A key issue in HNSCC pathogenesis is their development within large preneoplastic fields of mucosal epithelium made up of genetically altered cells that are clonally related to the carcinoma. Other individual differences in development of HNSCC comprise infection with pathogenic microbes and oncogenic human papillomavirus (HPV) subtypes like HPV 16 (a strong risk factor mainly for oropharyngeal cancer). Interestingly, the presence of HPV 16 also goes in line with better outcome after therapy. Vaccination against HPV infection in children plays an increasing role in prevention strategies and probably also reduces the oncogenic risk for HNSCC.
\end{abstract}

Keywords Head and neck squamous cell carcinoma . HNSCC · HPV · HPV vaccination · Cancer susceptibility . Genetic repair - Enzyme polymorphisms · Short term culture assay

A. Dietz is a National Representative of EPMA in Saxony, Germany.

A. Dietz $\cdot$ G. Wichmann

Department of Otolaryngology, Head and Neck Surgery,

University Clinic of Leipzig,

Leipzig, Germany

A. Dietz $(\bowtie)$

Klinik und Poliklinik für Hals-, Nasen-,

Ohrenheilkunde/Plastische Operationen, Universität Leipzig,

Liebigstrasse 10-14,

Leipzig 04103, Germany

e-mail: andreas.dietz@medizin.uni-leipzig.de

URL: http://www.uni-leipzig.de/ hno/

\section{Introduction}

Cancer of the head and neck covers a brought spectrum of different entities located in the upper digestive and respiratory tract. The organs addressed are larynx, pharynx (including naso-, oro- and hypopharynx), the oral cavity, scull base, thyroids and the salivary glands. This article focuses on squamous cell carcinomas of the larynx, pharynx and oral cavity which cover the majority of nearly $90 \%$ of the head and neck carcinomas (HNSCC).

The individual susceptibility to develop a HNSCC depends not only on the genetic background of the patient but mostly is triggered by a number of factors contributing to an impaired health status of the epithelia in the oral cavity but the pharynx and the larynx as well. Smoking and alcohol consumption are main factors damaging the normal physiology of squamous epithelia and their function and therefore are the main factors for the development of HNSCC. It was shown by numerous authors (e.g. [1, 2]) that risk for HNSCC is directly correlated with duration of smoking and number of cigarettes smoked, and considerably increased in individuals consuming more than $50 \mathrm{~g}$ of alcohol per day. Of particular interest is the synergism of both risk factors and the observation that the number of pack years number of years smoking multiplied with the mean number of cigarettes per day divided by 20 , the usual pack size) best correlates e.g. with an increasing risk for development of laryngeal HNSCC $[1,2]$ seemingly independent from the actual time period of smoking.

It is well known, that persistence of any irritation and inflammation increases the risk of cancer. The irritating and pro-inflammatory effects of tobacco smoke as well as those of higher concentrated alcohol and other irritants like those of chemical irritants or esophageal reflux not only trigger 
inflammation but also interfere with a proper voice and cause dysfunction of the epithelia e.g. of the vocal chords and lead to lesions and increased need for proliferation and repair.

In addition to tobacco smoke and alcohol abuse, other factors are able to increase the risk for HNSCC and thus should be prevented. These life-style related factors include improper hygiene like reduced numbers of tooth brushes but also high-frequent use of aggressive fluids for mouthwashes and gargling can disturb the normal mouth flora and do harm to the epithelia. This reduces their barrier function and, hence, makes them more vulnerable for infection with pathogens. However, the need to replace damaged cells and to repair small mucosal lesions contributes to an increased vulnerability due to the need for higher mitosis rate and proliferation, which increase the general risk for genetic instability and accumulation of somatic mutations. The latter leads to hyperplasia and, later on, often further to the development of leukoplakia, preneoplastic fields of mucosal epithelium called SIN (squamous intraepithelial neoplasia) and their progression from stage SIN I over SIN II to SIN III.

The development of SIN III (which reflects the earliest stage of detectable breakthrough of the malignant cells through the basal membrane) is the key issue in HNSCC pathogenesis. HNSCC commonly develop within large preneoplastic fields of SIN I to III which are made up of genetically altered cells that are clonally related to the carcinoma. These preneoplastic fields often extend into the surgical margins when tumours are excised, and can cause local recurrences and second primary tumours [3, 4]. In the recent years more knowledge about individual biologic factors of the given tumour and the individual patient, suffering from the tumour has been generated. Not only the physician treating the disease but also the whole scientific community which is concerned with head and neck cancer has to face more to implicated multimodality treatment options for HNSCC also suggested as alternative treatment to surgery even in resectable disease. In many cases, selecting the right treatment is difficult due to the lack of valid predictive markers to achieve some information on the individual response to the single treatment option. Regarding chemo radiation, difficulties such as early and late toxicities $[5,6]$, reduced functional outcome, and treatment failure leading to high risk salvage surgery with severe complications in many cases have to be managed. To address this problem, Lefebvre and Ang [7] worked out a list of guidelines for better outcome-specification after organ preservation chemo radiation, which should be used in future clinical trials. These recommendations defined a new endpoint: laryngoesophageal dysfunction-free survival. Also considering better response (i.e. results), future trial populations should include patients with limited tumour size like T2 or T3 laryngeal or hypopharyngeal squamous cell carcinoma taking into account additional specific factors. Following the recommendations of Lefebvre and Ang [7], biomarker studies including excision repair crosscomplementary-1 gene, E-cadherin, beta-catenin, epiregulin, amphiregulin, and p53 mutation should be favoured aiming at the enthusiastic goal of finding hints for individual treatment recommendations. Furthermore, chemo resistance of tumour cells in general can be provoked by mutations in oncogenes (e.g., K-ras), loss of tumour suppressors (e.g., p53, p16 ${ }^{\text {INK4a }}$ ) or dysregulation of genes involved in cell cycle control, cell proliferation, signal transduction, angiogenesis or apoptosis [8]. Focusing on the update discussion of molecular biological relevance in HNSCC by Leemans and colleagues, limitless replicative potential of head and neck cancer cells is caused by abrogation of the p53 and retinoblastoma (RB) pathways that perturb cell cycle regulation, probably in the context of telomerase reverse transcriptase (TERT) expression and under influence of the expressed HPV 16 proteins E6 and E7 [3]. A subgroup of HNSCCs becomes independent from growth factors owing to somatic changes in the epidermal growth factor receptor (EGFR) signalling pathway. HNSCC escape from the growth inhibitory transforming growth factor- $\beta$ (TGF $\beta$ ) pathway by somatic mutation or chromosomal loss of key genes. This pathway seems to be interconnected to the nuclear factor- $\mathrm{kB}(\mathrm{NF}-\mathrm{kB})$ pathway. Furthermore, the PI3KPTEN-AKT pathway is frequently activated in HNSCC, wherefore it might be a target in new specific treatment strategies [3].

Unfortunately in HNSCC, all these biomarkers showed vague correlation with better outcome after chemo radiation, but suffer from strongly discriminating results, many technical peculiarities, and in particular from investigations being addressed only in highly selected artificial surrogates for real tumours, namely cell lines; thus the clinical relevance is still limited (except regarding $\mathrm{p} 16^{\mathrm{INK} 4 \mathrm{a}}$ and HPV 16 in oropharyngeal cancer) [9] and so far are not suitable for individualised or personalised decision making. Past and current literature in translational research focuses mainly on laryngeal and, since HPV 16 came up, in particular on oropharyngeal HNSCC.

Regarding prevention strategies and as well the move towards personalised medicine in head and neck oncology, this article will give some ideas about the different individual factors of susceptibility, genetic alterations due to mutagens and virus infections, and discuss new methods of ex vivo performed pre-treatment response evaluation, which could be taken into account for future decision making. 


\section{Environmental risk factors}

The main risk factors for HNSCC are the lifestyle factors tobacco smoking and alcohol consumption [1], while there are also occupational substances like asbestos, polycyclic aromatic hydrocarbons, dust and solvents, promoting the development of HNSCC $[2,10,11]$. There is some evidence for an influence of genetic factors, as people with a family history of head and neck cancer were shown to have an increased risk of developing laryngeal cancer [12]. Despite this knowledge, it is not well known up to which extent survival time after diagnosis of laryngeal cancer is influenced by the above mentioned factors [13]. Cancer risk is directly correlated with duration of smoking and number of cigarettes smoked, and considerably increased in individuals consuming more than $50 \mathrm{~g}$ of alcohol per day. Tobacco smoke contains many carcinogens including a group of N-nitrosamines that produce carcinogenic methyl and pyridyloxobutyl DNA adducts [14]. A further constituent of tobacco smoke is the highly toxic acetaldehyde that produces genotoxic 1,N2-propano-20-deoxyguanosine DNA adducts. Acetaldehyde is also an intermediate product of ethanol metabolism and its concentration increases in a multiplicative manner in individuals who are simultaneously smoking and drinking alcohol [14]. This observation might explain the synergistic and multiplicative effect found for alcohol and smoking in laryngeal cancer risk and augments the important aspect of confounding by tobacco and alcohol in HNSCC epidemiologic research [2].

\section{Genetic factors of individual susceptibility}

Individual defence against mutagens

Obviously, the upper mentioned exogenous risk factors alone cannot, however, completely explain all cases of HNSCC. Therefore, many studies have been conducted to identify biomarkers that reflect host susceptibility more closely. Those that have been found include variations in carcinogen metabolism, DNA adduct formation and DNA repair activity [15]. The specific influences of these markers have been established for environmentally induced cancers such as lung tumours and HNSCC [16]. An important reaction in DNA repair pathways is poly(ADP-ribosyl)ation, which is induced by the presence of DNA single-strand breaks [15]. DNA damage associated with oxidative stress activates DNA repair pathways that involve various enzymes, including poly (ADP-ribose) polymerase (PARP) [17]. PARP is a highly conserved, ubiquitous nuclear enzyme found in most eukaryotic cells. Therefore, PARP activation is an immediate cellular response to metabolic, chemical, or radiation-induced DNA single strand breaks, reflecting the main mutational mechanism in development of HNSCC.

Several forms of cancer are more dependent on PARP than regular cells, making PARP an attractive target for chemotherapeutic cancer therapy. Therefore, a new group of PARP-inhibitors has been developed and is under clinical investigation in lung, prostate, colorectal and breast/ovarian cancer. Some cancer cells that lack the tumour suppressor PTEN may be sensitive to PARP. Hence PARP inhibitors may be effective against many PTENdefective tumours (e.g. some aggressive prostate cancers and assumable HNSCC) [8].

A unique and for detection of individual susceptibility very interesting property of PARP is that it is inactive unless DNA single strand breaks are present [18]. Poly(ADP-ribose) undergoes degradation, which is catalysed by poly(ADP-ribose) glycohydrolase [19] resulting in rapid turnover of the polymer under conditions of DNA breakage. Poly(ADP-ribosyl)ation plays a major role in facilitating DNA base excision repair and in maintaining the genomic stability of cells exposed to genotoxic stress [16] and might have a significant impact on the process of neoplastic transformation. This assumption is based on findings that defective DNA repair is associated with increased risks for cancer in humans and that inter-individual differences in DNA repair capacity are markers for individual susceptibility to cancer [20].

We investigated whether differences in the activity of PARP are associated with the risk for laryngeal cancer within the so called Rhein-Neckar-Larynx trial [15]. In this case-control study on genetic, lifestyle and occupational risk factors for laryngeal cancer, PARP activity was assessed as DNA damage-induced poly(ADP-ribose) formation in human peripheral blood lymphocytes. Polymer formation was determined as the cellular response to bleomycin, a well-known inducer of DNA strand breaks, in lymphocytes from 69 laryngeal cancer patients and 125 healthy controls. The frequency of bleomycin-induced polymer formation, measured as mean pixel intensity, was significantly lower in cases $(74.6, \mathrm{SE}=3.7)$ than in controls (94.5, $\mathrm{SE}=3.5$ ), and was not influenced by smoking, age or sex. There was no significant difference between cases (59.1, $\mathrm{SE}=5.2)$ and controls (50.5, $\mathrm{SE}=3.7)$ in basal polymer formation (in cells not treated with bleomycin). When the upper tertile of polymer formation was used as the reference, the odds ratio for the lower tertile of bleomycin induced polymer formation was $3.79(95 \%$ confidence interval $=95 \% \mathrm{CI}: 1.37-10.47, p=0.01$ ). Peripheral blood lymphocytes from laryngeal cancer patients thus showed significantly less bleomycin-induced 
poly(ADP-ribose) formation. These results suggest that a reduced PARP activity might be associated with an increased risk for laryngeal cancer.

There are more than 150 human DNA repair genes other than PARP known [21]. These can be sub-grouped into genes associated with DNA damage signalling and regulation of the repair system genes and genes working with distinct repair pathways such as mismatch repair, base excision repair, nucleotide excision repair (NER), direct damage reversal, and DNA double-strand break repair. NER is the main pathway for removing the bulky DNA adducts caused by the chemical mutagens present in tobacco smoke and alcohol. There are about 30 proteins involved in NER [22]. NER consists of two sub-pathways, namely the global genome repair (GGR) that repairs anywhere in the genome, and the transcription coupled repair (TCR) involved in damage repair at actively transcribing sites. The complex interaction of the NER proteins in the pathway is not yet completely known, but damage recognition is specific for each of these sub-pathways (more details in [21]). As already mentioned, laryngeal cancer is known to be associated with smoking and high alcohol consumption. Nucleotide excision repair (NER) plays a key role in repairing DNA damage induced by these exposures, and hence might affect laryngeal cancer susceptibility. In the upper mentioned Rhein-Neckar-Larynx trial that included 248 cases and 647 controls, the association of laryngeal cancer with 14 single nucleotide polymorphisms (SNPs) in eight NER genes (XPC, XPA, ERCC1, ERCC2, ERCC4, ERCC5, ERCC6, and RAD23B) was analysed with respect to smoking and alcohol exposure. Data were stratified for age and gender, and adjusted for smoking, alcohol consumption and education. Pro-carriers of ERCC6 Arg1230Pro showed a decreased risk for laryngeal cancer (OR 5 0.53, 95\% CI: 0.34-0.85), strongest in heavy smokers and high alcohol consumers. ERCC5 Asp1104His was associated with risk in heavy smokers (OR 5 1.70, 95\% CI: 1.1-2.5). Val-carriers of RAD23B Ala249Val had an increased cancer risk in heavy smokers (OR 5 1.6, 95\% CI: 1.1-2.5) and high alcohol consumers (OR 5 2.0, 95\% CI: 1.1-3.4). The combined effect of smoking and alcohol intake affected risk, at high exposure level, for ERCC6 1230Pro carriers (OR 5 0.47, 95\% CI: 0.22-0.98) and RAD23B 249Val carriers (OR 5 2.6, 95\% CI: 1.3-4.9). When tested for gene-gene interaction, presence of three risk alleles in the XPC-RAD23B complex increased the risk 2.1-fold [21].

The data strengthen the evidence that common genetic variations in NER genes can significantly modify HNSCC risk. However, until now it is not yet completely clear if this knowledge might add to prevention strategies or even decision-making regarding selection of optimised multimodal treatment regimens.
So far it has to be summarised, that only little numbers of investigations in the field of genetic susceptibility suggest existence of defined inter-individual differences in genetic defence against mutagens in patients which could be considered to be useful for further additional personalisation of treatment strategies for an individual HNSCC.

Differences in detoxification of hazardous substances

Some previous studies give important hints to genetic polymorphisms in alcohol dehydrogenases (ADH) as risk modifiers for head and neck cancer. ADH1B and ADH1C (previously called $\mathrm{ADH} 2$ and $\mathrm{ADH} 3$ respectively) are responsible for the metabolism of ethanol to acetaldehyde, which is classified as potentially carcinogenic to humans [23]. Higher ethanol oxidation activity has been associated with the ADH1B_2 allele however, a 6.6- fold increased risk of head and neck cancer has been observed in homozygous carriers of the ADH1B_1 allele among Japanese alcoholics [24]. In the case of ADH1C, the presence of the _1 allele results in a higher activity in alcohol metabolism capacity and formation of acetaldehyde than the 2 allele. Thus, ADH1C_1 has been implicated as a risk factor for laryngeal tumours. Indeed, this was shown in one small study on 39 alcoholic men with upper aerodigestive tract cancer, including 18 with laryngeal tumours, compared to 37 alcoholic male controls, but so far was not confirmed in other studies investigating head and neck cancers risk in Caucasians [14]. As reported in an abstract, a German study showed an increased risk of ADH1C 1 carriers for tumours of the upper alimentary tract, as well as higher salivary acetaldehyde concentrations in volunteers with the ADH1C_1_1 genotype [14].

Glutathione-S-transferases (GST) M1 and T1 are involved in the detoxification of tobacco carcinogens previous studies concerning the relevance of the highly prevalent homozygous deletion of the genes (GSTM1 null or GSTT1 null, respectively) to risk of head and neck cancer have yielded inconsistent results. The data of the Rhein-Neckar-Larynx trial revealed no significance regarding the specific enzyme polymorphisms in HNSCC. Neither the putative risk genotypes ADH1B 2/_1 (OR 0.86, 95\% CI: $0.41-1.82$ ) or ADH1C_1/ 1 (OR 1.06, 95\% CI: 0.7-1.62) nor GSTM1 null (OR 0.94 , 95\% CI: $0.62-1.42$ ) or GSTT1 null (OR $1.34,95 \%$ CI: 0.74-2.42) were associated with an overall increased risk for laryngeal cancer even in stratified analysis [14]. The lack of risk modification by the studied genotypes emphasises the ethnic differences between Caucasians and the Asian (Japan) population and points to ethnic different patterns of HNSCC susceptibility. 


\section{HPV 16 as risk and predictive factor for oropharyngeal cancer}

Growing relevance has been demonstrated for the impact of infection with oncogenic subtypes of the human papilloma virus (HPV), and in particular presence of HPV 16 in and its influence on development of HNSCC. Although HPV 16 alone accounts for more than $90 \%$ of cases of HPVpositive squamous-cell carcinomas of the oropharynx, a more accurate and probably higher proportion might be found by testing for other types of HPV (e.g., types 18, 31, 33 , and 35), which are infrequently detected in oropharyngeal cancers. Genomic DNA of oncogenic HPV is detected in approximately $26 \%$ of all squamous-cell carcinomas of the head and neck worldwide but the molecular evidence is most rigorous and consistent for oropharyngeal squamouscell carcinoma, in which viral integration and the expression of viral oncogenes (E6 and E7) have been shown [25]. The example of the relationship between HPV and cervical cancer [26] indicates that high-risk sexual behaviour will increase the risk of other cancers caused by HPV [27]. In the remarkable report by $\mathrm{D}^{\prime}$ Souza could be demonstrated that sexual behavior (oral sex, high lifetime numbers of sex-partners) correlates with a higher incidence of oral HPV 16 infection. Furthermore, HPV 16 presence correlates significantly with elevated risks of oropharyngeal cancer independent to degree of tobacco and alcohol abuse [27]. Several retrospective case series have shown that among patients with oropharyngeal HNSCC, patients with HPVpositive tumours have a better prognosis than patients with HPV-negative tumours [25]. The recently published retrospective study of the RTOG provides strong evidence that tumour HPV status is an independent prognostic factor for overall survival and progression-free survival among patients with oropharyngeal squamous-cell carcinomas, which is consistent with the hypothesis that HPV-positive and HPV-negative oropharyngeal squamous-cell carcinomas are distinct and have different causes, risk-factor profiles [28] and survival outcomes. Ang and co-authors could demonstrate clearly, that patients suffering from oropharyngeal carcinoma with negative HPV 16 stage and high tobacco intake are at highest risk compared to the group of HPV 16-positive, non smoking patients [29]. Due to this overwhelming difference, the authors recommend to separate HPV 16 associated tumours in future clinical trials. Moreover, the existing data suggest that HPV 16 is a strong marker which could be used and should lead to differentiated treatment strategies due to better radio- and chemo response in the HPV 16-positive group. Whether patients with HPV-positive tumours who are considered to be in the low-risk category can be spared the long-term complications of intensive, multimodal therapy without compromising their survival is now a highly relevant clinical question
[28]. So HPV 16 is a new marker for personalised treatment concepts in HNSCC.

\section{Vaccination against oncogenic HPV subtypes as appropriate predictive action}

Two vaccines are available for vaccination against the two high-risk HPV subtypes 16 and 18: Gardasil (developed by Merck Sharp \& Dohme Ltd. and Sanofi Pasteur in collaboration with the German Cancer Research Center [DKFZ] in Heidelberg), and Cervarix (GlaxoSmithKline). Gardasil contains genetically engineered capsid proteins of HPV 16 and 18 and also of the low-risk subtypes 6 and 11, which are absent in Cervarix. Gardasil is approved for vaccination in the EU since 2006 for vaccination of females (age 9-26) and boys (age 9-15). Cervarix is a bivalent recombinant vaccine for the prevention of cervical precancers and cervical cancer associated with HPV 16 and HPV 18 only approved by the EMEA (in 2007) and FDA (in 2009) for use in girls and young women (aged 10-25). Regarding vaccination efficacy, the view so far was focused on cervix carcinoma [30]. Since both vaccines have efficacy against, and disease contribution of, HPV 16, HPV 18, but also other oncogenic HPV types not included in the vaccine (e.g. HPV 31), their use might allow for a reduction of HPV-induced cancers not only of the cervix. However, vaccination of girls and young females and also of boys against the oncogenic HPV subtypes might not only add to prevention of this malignancy by prevention of transmission of HPV as causative agents of cervix cancer but also of other cancer including HNSCC [30-32].

\section{Markers for individual response evaluation}

Despite ASCO in 2004 admonished from assay based therapy in general outside of clinical trials [33], the field of clonogenic and colony-formation assays for chemosensitivity testing developed encouraging new directions in various tumour entities. Individual response evaluation for better biology-based decision making is more desirable than ever since various competitive therapy strategies even in case of resectable HNSCC (i.e. advanced laryngeal and hypopharyngeal cancer) are increasingly promoted, and many new substances, antibodies, and small molecules come into the clinical view. Especially in multimodality treatment in current clinical trials aiming on inclusion of pharmaceuticals with non-overlapping toxicity profiles and hence trying to execute treatment with escalating doses to fathom out the limits of maximum tolerable treatment regimes for selected patients with good general state of health we have to face dramatically increased early and late 
toxicities, which require more robust pre-treatment criteria to select responders (and non-responders for definitive surgical treatment). For HNSCC, assay-based individual testing was inflicted with a series of technical problems and principal questions like micro heterogeneity, flavin-induced cell toxicity, chaotropism due to photoreactivity of pharmaceuticals and presence of UV and other short-wavelength light, lack of differentiation between tumour and stromal cells, differences in chemosensitivity of tumour and stromal cells, structural needs for specific handling of the testing, lack of specific dose related sensitivity towards single and combined substances etc., but these issues have been addressed in recent publications (Overview in [34]). Currently, we emphasise the advantages of the FLAVINOassay for clinical research, which considers the above mentioned major pitfalls in assay-based diagnosis before starting the therapy. Unfortunately, this test requires a strong definitive hospital setting with a closed relation between the surgical and the laboratory units and performance under flavin-protecting conditions in a "same day" procedure. Besides further evaluation of definitive clinical introduction of assay-based guidance in decision-making for therapy additional, translational genetic and molecular biologic response marker research could be strongly pushed forward by correlating these results with the outcome in exvivo chemosensitivity testing of the individual tumour [35]. Figs. 1 and 2 show the correlation of response of the patient's tumour in vivo to one single cycle of induction chemotherapy with TPF (docetaxel, cisplatin, 5-fluorouracil) + cetuximab and the reduction of colony formation in the FLAVINO-assay in response to maximum tolerable plasma concentrations of the pharmaceuticals [36, 37].

It is mandatory that ex-vivo chemosensitivity testing in all settings of clonogenic and colony-formation assays should include all different cell types within a given tumour and being able to test these cells separately. Furthermore, detailed information about combination efficacy of different drugs and radiation therapy for the given tumour has to be addressed. In this respect, the FLAVINO-Assay indeed allows for a detailed analysis of colony formation by epithelial and stromal cells under exposure to either cisplatin or docetaxel and combined exposure to both. We think that ex-vivo chemosensitivity testing in HNSCC has reached a mature technical level and is feasible for use within clinical trials.

We go in line with the ASCO recommendation in 2004 [33] that chemosensitivity testing should not be used uncritically without selection of the right method outside of clinical trials. All clonogenic test systems which were propagated before 2004 failed to show suitability for proper chemo-sensitivity testing of HNSCC due to a number of reasons. Most relevant is the lack of flavin-protecting conditions, lack of separation between the different cell types, and mainly measurement only of sum signals which in general do not allow for more distinguished chemo recommendations. But, regarding personalised medicine as labelled by ASCO in 2009, test systems for HNSCC are promising since they have become more reproducible and differentiated in the recent years. The authors, for instance, obtained very promising results in the FLAVINO-assay [35]. These data underline that even in organ preservation treatment in advanced laryngeal cancer, predictive assays for better decision making in balancing competing therapy options is strongly needed since many patients still do not respond to multimodality treatment and have to go through salvage surgery procedures with high complication rates and severe late toxicity events, reducing significantly quality of life. In non responders, definitive surgery is the best treatment and should be favoured by enthusiastic research in more specific biology based pre-treatment diagnostics.

Chemosensitivity testing using the FLAVINO-assay could be an option for that and should be included into future clinical trials. A close and confiding cooperation with the pathologist is mandatory because of test material has to be fresh, unfixed, and must be quickly handled under flavin-protecting conditions. Furthermore, the FLAVINOassay can be recommended for pharmacological ex-vivo characterisation of new substances potentially useful in treatment of $\mathrm{HNSCC}$, proposing more realistic results
Fig. 1 Image series of a 71 years old male patient suffering from a laryngeal $\mathrm{cT} 4_{\mathrm{a}} \mathrm{cN} 2{ }_{\mathrm{b}} \mathrm{cM} 0$ carcinoma (left) pre and intra therapy after receiving one cycle TPF + cetuximab (right); the patient was early evaluated as good responder
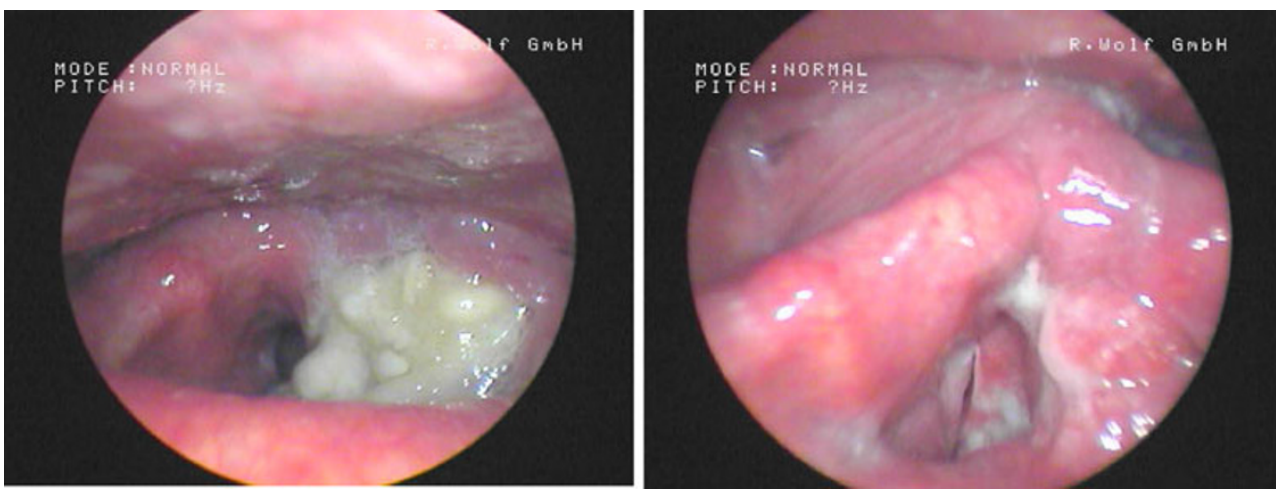
a

Colony formation:

$\mathrm{IC}_{50}$ of cisplatin [nM]

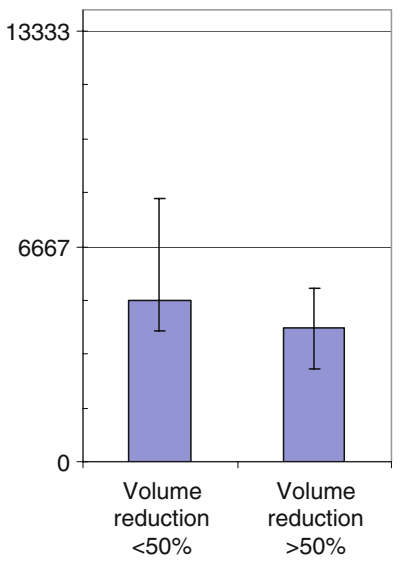

b Colony formation:

$\mathrm{IC}_{50}$ of docetaxel $[\mathrm{nM}]$

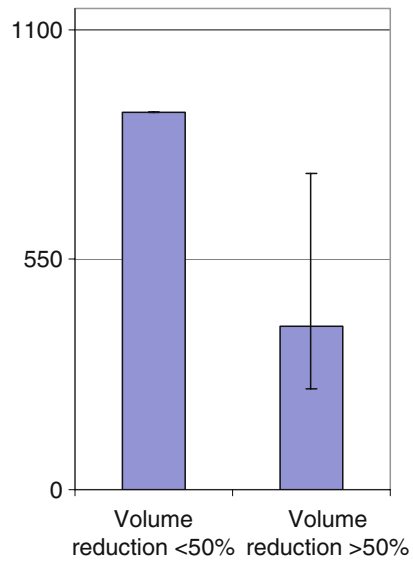

C
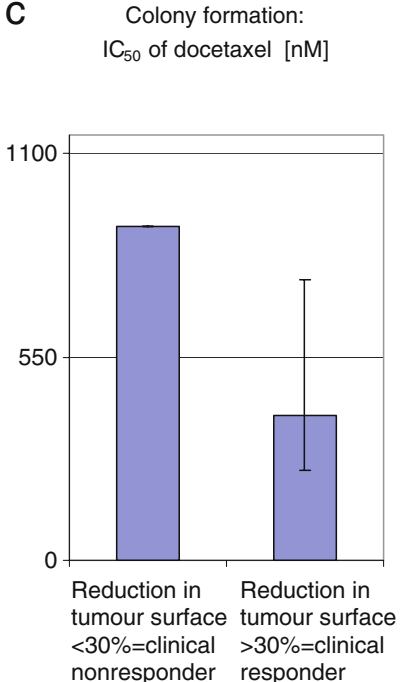

d

Colony formation:

$\mathrm{IC}_{50}$ of cisplatin $[\mathrm{nM}]$

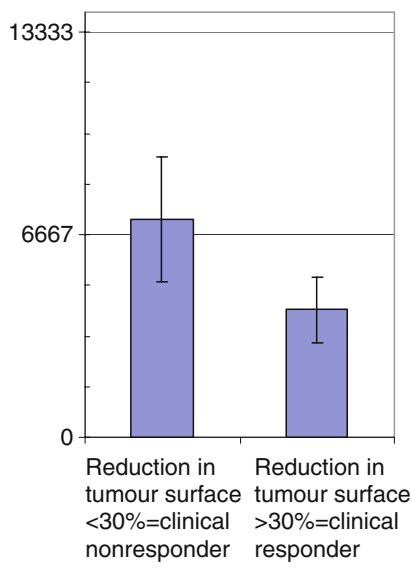

Fig. 2 Comparison of $\mathrm{IC}_{50}$ concentrations (inhibitory concentration which diminish colony formation to 50\%) in the FLAVINO assay [35] of HNSCC with lower response to TPF-IC with good responders. (a), (b): based on volume reduction $<50 \%$ (left) vs. $>50 \%$ (right); (c), (d): based on tumour-surface reduction $<30 \%$ vs. (left) vs. $>30 \%$ (right). Horizontal lines in a, c and b, d, represent maximum tolerable plasma

compared to animal models or established cell lines which are almost not representative for the average HNSCC. In this context, new developments like the TSC assay (tissue slice culture assay) [38] showed to be very promising since the architecture of the tissue can be preserved in culture. This method was first performed in brain tissue for investigation of pharmacological effects ex vivo. By use of living slices of $300 \mu \mathrm{m}$ thickness obtained from brain resections of four patients with pharmacoresistant temporal-lobe epilepsy, cell death was induced by tumour-necrosis-factor-related apoptosis-inducing ligand (TRAIL) [38]. Remarkably, this TRAIL-induced cell death revealed an important species difference between man and mouse which are widely used for preclinical studies: TRAIL did not damage murine brain cells. An important consequence of this finding was the definitive stop of further clinical phase-I trials with TRAIL to avoid fatal brain damage in patients. In future, development of this TSC-assay into ex-vivo testing of HNSCC and combining this approach with integration of the methodological advantages of the FLAVINO-assay could be an important advancement.

\section{Conclusions and outlook}

Personalised medicine becomes also relevant in decision making for treatment in HNSCC. Current therapy options in daily routine are driven by personal experience and a small number of clinical randomised trials, overestimating levels of cisplatin (6,667 $\mathrm{nM} \mathrm{[36])} \mathrm{and} \mathrm{docetaxel} \mathrm{(550} \mathrm{nM} \mathrm{[37]),}$ respectively. Clear differences in $\mathrm{IC}_{50}$ for $\mathrm{P}$ and $\mathrm{T}$ exist between responders and no responders, and these allow for their distinction and hence could build a rational to analyse chemo response before starting chemotherapy

the impact of chemo radiation due to the lack of well planned and conducted surgical trials. Future clinical trials should pay more attention to the individual biology/genetic background of the patient and the tumour including its vulnerability due to genetic susceptibility and life-style factors, presence of HPV 16 infection and other possible biomarkers so far not validated. These factors should not abandon the possible advantages of pre-treatment responseevaluation using the newly improved short term culture assays. Due to the fact that overall survival of patients suffering from HNSCC is still only $42 \%$ in Europe [39] and many of them underwent average treatment, which might be wrong for the single patients, there is still a lot of work to do in better canalising personalised concepts.

However, the better alternative to treatment in general should be prevention of the disease. The best prevention strategy for HNSCC is complete avoidance of exposure to tobacco smoke during the whole life time and also reduction of harm for the epithelia by avoidance of other self-induced exposure to irritating and mutagenic life styleassociated risk factors like alcohol and alcohol abuse. Further lowering of environmental and occupational exposure to irritating compounds and other groups of chemicals with mutagenic and immunomodulatory properties on the one side and proper oral hygiene and regular physical examination of the oral cavity by a dentist on the other side are also preventive against HNSCC or (referring to the latter) allows for early detection of precancerous lesions. In addition, vaccination against HPV appears to be a very attractive way to reduce the risk for HNSCC. 


\section{References}

1. Ramroth H, Dietz A, Becher H. Interaction effects and populationattributable risks for smoking and alcohol on laryngeal cancer and its subsites. A case-control study from Germany. Methods Inf Med. 2004;43(5):499-504.

2. Dietz A, Ramroth H, Urban T, Ahrens W, Becher H. Exposure to cement dust, related occupational groups and laryngeal cancer risk: results of a population based case-control study. Int J Cancer. 2004;108(6):907-11.

3. Leemans CR, Braakhuis BJ, Brakenhoff RH. The molecular biology of head and neck cancer. Nat Rev Cancer. 2011;11(1):922.

4. Roesch-Ely M, Leipold A, Nees M, Holzinger D, Dietz A, Flechtenmacher C, et al. Proteomic analysis of field cancerization in pharynx and oesophagus: a prospective pilot study. J Pathol. 2010;221(4):462-70.

5. Machtay M, Moughan J, Trotti A, Garden AS, Weber RS, Cooper JS, et al. Factors associated with severe late toxicity after concurrent chemoradiation for locally advanced head and neck cancer: an RTOG analysis. J Clin Oncol. 2008;26 (21):3582-9.

6. Rudat V, Eckel H, Volling P, Schröder M, Staar S, Wallner F, et al. Long-term results of a prospective multicenter phase II study to preserve the larynx function using concomitant boost radiochemotherapy with Carboplatin. Radiother Oncol. 2008;89 (1):33-7.

7. Lefebvre JL, Ang KK. Larynx Preservation Consensus Panel. Larynx preservation clinical trial design: key issues and recommendations-a consensus panel summary. Int J Radiat Oncol Biol Phys. 2009;73(5):1293-303.

8. Domagala P, Huzarski T, Lubinski J, Gugala K, Domagala W. PARP-1 expression in breast cancer including BRCA1-associated, triple negative and basal-like tumors: possible implications for PARP-1 inhibitor therapy. Breast Cancer Res Treat. 2011; doi:10.1007/s10549-011-1441-2

9. Dietz A, Boehm A, Mozet C, Wichmann G, Giannis A. Current aspects of targeted therapy in head and neck tumors. Eur Arch Otorhinolaryngol. 2008;265 Suppl 1:3-12.

10. Becher H, Ramroth H, Ahrens W, Risch A, Schmezer P, Dietz A. Occupation, exposure to polycyclic aromatic hydrocarbons and laryngeal cancer risk. Int J Cancer. 2005;116(3):451-7.

11. Ramroth H, Dietz A, Ahrens W, Becher H. Occupational wood dust exposure and the risk of laryngeal cancer: a population based case-control study in Germany. Am J Ind Med. 2008;51(9):64855 .

12. Garavello W, Turati F, Bosetti C, Talamini R, Levi F, Lucenteforte $\mathrm{E}$, et al. Family history of cancer and the risk of laryngeal cancer: a case-control study from Italy and Switzerland. Int J Cancer. 2011; doi:10.1002/ijc.26055

13. Rudolph E, Dyckhoff G, Becher H, Dietz A, Ramroth H. Effects of tumour stage, comorbidity and therapy on survival of laryngeal cancer patients: a systematic review and a meta-analysis. Eur Arch Otorhinolaryngol. 2011;268(2):165-79.

14. Risch A, Ramroth H, Raedts V, Radjaee-Behbahani N, Schmezer $\mathrm{P}$, Becher $\mathrm{H}$, et al. Genetic Polymorphisms in class I alcohol dehydrogenases $\mathrm{ADH} 1 \mathrm{~B}$ and $\mathrm{ADH} 1 \mathrm{C}$, and glutathione-Stransferases GSTM1 and GSTT1 do not significantly modify the alcohol- and tobacco smoke-associated laryngeal cancer risk. Pharmacogenetics. 2003;13(4):225-30.

15. Rajaee-Behbahani N, Schmezer P, Ramroth H, Bürkle A, Bartsch $\mathrm{H}$, Dietz A, et al. Reduced poly(ADP-ribosyl)ation in lymphocytes of laryngeal cancer patients: results from a case-control study. Int J Cancer. 2002;98(5):780-4.
16. Cloos J, Braakhuis BJ, Steen I, Copper MP, de Vries N, Nauta JJ, et al. Increased mutagen sensitivity in head-and-neck squamouscell carcinoma patients, particularly those with multiple primary tumors. Int J Cancer. 1994;6:816-19.

17. Walisser JA, Thies RL. Poly(ADP-ribose)polymerase inhibition in oxidant-stressed endothelial cells prevents oncosis and permits caspase activation and apoptosis. Exp Cell Res. 1999;251:401-13.

18. Bürkle A. Poly(ADP-ribosyl)ation, a DNA damage-driven protein modification and regulator of genomic instability. Cancer Lett. 2001;163:1-5.

19. Miwa M, Tanaka M, Matsushima T, Sugimura T. Purification and properties of a glycohydrolase from calf thymus splitting riboseribose linkage of poly(adenosine diphosphate ribose). J Biol Chem. 1974;249:3475-82.

20. Berwick M, Vineis P. Markers of DNA repair and susceptibility to cancer in humans: an epidemiological review. J Natl Cancer Inst. 2000;92:874-97.

21. Abbasi R, Ramroth H, Becher H, Dietz A, Schmezer P, Popanda O. Laryngeal cancer risk associated with smoking and alcohol consumption is modified by genetic polymorphisms in ERCC5, ERCC6 and RAD23B but not by polymorphisms in five other nucleotide excision repair genes. Int J Cancer. 2009;125(6):14319.

22. Andressoo JO, Hoeijmakers JH, Mitchell JR. Nucleotide excision repair disorders and the balance between cancer and aging. Cell Cycle. 2006;5:2886-8.

23. IARC. Re-evaluation of some organic chemicals, hydrazine and hydrogen peroxide (part two), 319-335. IARC Monographs on the evaluation of the carcinogenic risk of chemicals to humans (71). Lyon, IARC, 1999.

24. Yokoyama A, Muramatsu T, Omori T, Yokoyama T, Matsushita S, Higuchi S, et al. Alcohol and aldehyde dehydrogenase gene polymorphisms and oropharyngolaryngeal, esophageal and stomach cancers in Japanese alcoholics. Carcinogenesis. 2001;22:4339.

25. Marur S, D'Souza G, Westra WH, Forastiere AA. HPV-associated head and neck cancer: a virus-related cancer epidemic. Lancet Oncol. 2010;11(8):781-9.

26. Bosch FX, De Sanjose S. Human papillomavirus and cervical cancer - burden and assessment of causality. J Natl Cancer Inst Monogr. 2003;31:3-13.

27. D'Souza G, Kreimer AR, Viscidi R, Pawlita M, Fakhry C, Koch WM, et al. Case-control study of human papillomavirus and oropharyngeal cancer. N Engl J Med. 2007;356(19):1944-56.

28. Gillison M, D’Souza G, Westra W, Sugar E, Xiao W, Begum S, et al. Distinct risk factor profiles for human papillomavirus type 16positive and human papillomavirus 16-negative head and neck cancers. J Natl Cancer Inst. 2008;100:407-20.

29. Ang KK, Harris J, Wheeler R, Weber R, Rosenthal DI, Nguyen-Tân PF, et al. Human papillomavirus and survival of patients with oropharyngeal cancer. N Engl J Med. 2010;363 (1):24-35.

30. The FUTURE II Study Group. Quadrivalent Vaccine against Human Papillomavirus to Prevent High-Grade Cervical Lesions. N Engl J Med. 2007;356:1915-27.

31. Kim JJ. Weighing the benefits and costs of HPV vaccination of young men. N Engl J Med. 2011;364(5):393-5.

32. Giuliano AR, Palefsky JM, Goldstone S, Moreira Jr ED, Penny $\mathrm{ME}$, Aranda $\mathrm{C}$, et al. Efficacy of quadrivalent HPV vaccine against HPV Infection and disease in males. $\mathrm{N}$ Engl $\mathrm{J}$ Med. 2011;364(5):401-11. Erratum in: N Engl J Med. 2011; 364 (15): 1481.

33. Schrag D, Garewal HS, Burstein HJ, Samson DJ, Von Hoff DD, Somerfield MR. ASCO Working Group on Chemotherapy Sensitivity and Resistance Assays. American Society of Clinical 
Oncology Technology Assessment: chemotherapy sensitivity and resistance assays. J Clin Oncol. 2004;22(17):3631-38.

34. Dietz A, Boehm A, Horn IS, Kruber P, Bechmann I, Golusinski W, et al. Assay-based response evaluation in head and neck oncology: requirements for better decision making. Eur Arch Otorhinolaryngol. 2010;267(4):483-94.

35. Dietz A, Boehm A, Reiche A, Mueller G, Kruber P, Keilholz U, et al. Prediction of outcome of TPF with or without cetuximab induction chemotherapy in head and neck squamous cell carcinoma (HNSCC) using the FLAVINO assay. J Clin Oncol. 2010;28:15s, abstr. 5572.

36. Desoize B, Berthiot G, Manot L, Coninx P, Dumont P. Evaluation of a prediction model of cisplatin dose based on total platinum plasma concentration. Eur J Cancer. 1996;32A:17348.

37. Bissett D, Setanoians A, Cassidy J, Graham MA, Chadwick GA, Wilson P, et al. Phase I and pharmakokinetic study of Taxotere (RP 56976) administered as a 24-hour infusion. Cancer Res. 1993;53:523-7.

38. Nitsch R, Bechmann I, Deisz RA, Haas D, Lehmann TN, Wendling U, et al. Human brain-cell death induced by tumour-necrosis-factorrelated apoptosis-inducing ligand (TRAIL). Lancet. 2000;356 (9232):827-8

39. Sant M, Allemani C, Santaquilani M, Knijn A, Marchesi F. EUROCARE Working Group. EUROCARE-4. Survival of cancer patients diagnosed in 1995-1999. Results and commentary. Eur J Cancer. 2009;45(6):931-91. 